\title{
AS DIFICULDADES DE APRENDIZAGEM ESCOLAR SOB O OLHAR DA PSICANÁLISE: UMA INVESTIGAÇÃO DA PRODUÇÃO CIENTÍFICA
}

Sandra Fernandes de FREITAS ${ }^{1}$ Maria Lúcia de OLIVEIRA ${ }^{2}$

RESUMO: O trabalho apresenta uma investigação do estágio atual da produção científica acadêmica no período de 1996 a 2008, sobre a problemática de crianças que apresentam dificuldades de aprendizagem escolar tendo como base a teoria psicanalítica. Esta investigação insere-se no Projeto de Mestrado em Educação Escolar da Faculdade de Ciências e Letras da UNESP/Araraquara. Consideramos que a problemática relacionada à dificuldade de aprendizagem é multifatorial e o psiquismo está intimamente relacionado às condições de aprendizagem. As questões inspiradoras para esta pesquisa são o desejo de aprender/conhecer e o conceito que as crianças com dificuldades de aprendizagem têm de si mesmas. Com o objetivo de identificarmos o que vem sendo desenvolvido nesta área, buscou-se na base de dados da CAPES, através das palavras chaves: psicanálise e educação escolar, resumos de teses e dissertações. Dos 45 resumos selecionados, relacionados à temática em questão, 36 deles tratam o tema das dificuldades de aprendizagem sob a ótica do professor, de questões sócio-político-educacionais, dos programas de inclusão de crianças com necessidades educativas especiais ou teoricamente. Apenas 09 trabalhos incluem a criança como sujeito de investigação; 06 são estudos de caso e 03 utilizam-se de entrevistas e outros recursos para investigar o que as crianças enunciam sobre a sua problemática. Concluímos que a psicanálise tem sido utilizada enquanto teoria para abordar a educação e contribuir com novos olhares que considerem os aspectos psíquicos, implicados nas relações estabelecidas nesse campo. Entretanto, as pesquisas sobre dificuldades de aprendizagem que focalizam o sujeito aprendente são pouco representativas.

PALAVRAS-CHAVE: Psicanálise. Dificuldade de aprendizagem. Criança.

Este trabalho integra uma pesquisa de Mestrado em Educação Escolar da Faculdade de Ciências e Letras da UNESP/Araraquara. Trata-se de uma investigação sobre a problemática de crianças que apresentam dificuldades de aprendizagem escolar e tem como sustentação teórica a psicanálise. Consideramos que a problemática relacionada à dificuldade de aprendizagem é multifatorial e que o psiquismo está implicado nas condições de aprendizagem. As questões que nos inspiram nesta pesquisa dizem respeito ao desejo de

\footnotetext{
${ }^{1}$ Mestranda em Educação Escolar. UNESP- Universidade Estadual Paulista. Faculdade de Ciência e Letras Pós- Graduação em Educação Escolar - Centro de Pesquisas da Infância e da Adolescência "Dante Moreira Leite”.. Araraquara - SP - Brasil. 14800-901 - sff@fclar.unesp.br

${ }^{2}$ UNESP -- Universidade Estadual Paulista. Faculdade de Ciência e Letras - Departamento de Psicologia da Educação - Grupo de Pesquisa em Psicanálise e Educação. Araraquara - SP- Brasil. 14800-901 luciaegumar@yahoo.com.br
} 
aprender/conhecer e ao conceito que as crianças que apresentam dificuldades de aprendizagem têm de si mesmas.

A partir dessa temática, apresentaremos a seguir o estágio atual da produção científica acadêmica do período de 1996 a 2008 com o objetivo de identificarmos o que vem sendo desenvolvido nesta área, sob o foco da psicanálise.

Para cumprir tal objetivo, foi realizado um levantamento no site da Capes Coordenação de Aperfeiçoamento de Nível Superior de Teses e Dissertações num período de 12 anos que tratassem do tema psicanálise e dificuldade de aprendizagem, palavras que inicialmente foram utilizadas como palavras chave. Porém, através destas palavras foram encontradas poucas referências. Muitas vezes o resultado obtido era "nenhum título foi encontrado”, ou então, apenas uma ou duas referências eram indicadas em cada universidade pesquisada durante o período selecionado. Esse fato nos levou a trabalhar com uma palavra chave mais ampla - Educação Escolar. O uso das palavras chave: Psicanálise e Educação Escolar proporcionou um número ampliado de trabalhos, o que permitiu uma visão mais abrangente das pesquisas que estão sendo produzidas na interface da psicanálise com a educação escolar.

Foram pesquisadas as Universidades Públicas USP, UNESP e UNICAMP, as Universidades Federais de São Carlos, Rio de Janeiro e Rio Grande do Sul e também a Universidade particular PUC - Pontifícia Universidade Católica de Campinas, São Paulo, Rio de Janeiro e Rio Grande do Sul.

Para não descartarmos trabalhos que pudessem nos trazer contribuições, procuramos ao ler os resumos, selecionar aqueles que fizessem alguma referência às dificuldades escolares de forma geral. Com esse critério foram selecionamos 45 resumos considerando a possibilidade de alguma contribuição, seja teórica ou prática para o nosso trabalho.

De uma análise mais detalhada desses resumos, obtivemos os seguintes resultados: em 36 trabalhos, identificamos diferentes questões relacionadas às dificuldades de aprendizagem e que foram tratadas sob diferentes perspectivas: do professor e/ou de outros profissionais da escola, de questões sócio-político-educacionais, dos programas de inclusão, da família, ou ainda, que tratam do tema apenas sob o ponto de vista de pressupostos teóricos. Consideramos a relevância destes trabalhos, porém não serão aqui tratados por não integrarem a criança em suas pesquisas.

Encontramos apenas 09 trabalhos que incluem a criança como sujeito de investigação; 06 são estudos de caso e 03 utilizam-se de entrevistas e outros recursos para investigar o que as crianças enunciam sobre a sua problemática. 
Apresentaremos a análise dos 09 resumos que incluem a criança como sujeito de investigação e que estão mais diretamente relacionados à nossa temática.

Selecionamos estes trabalhos justamente por abordarem a questão da dificuldade de aprendizagem, porém são trabalhos que partem de diferentes problemáticas relacionadas às crianças. Desse modo, não se caracteriza por abordarem pontualmente os processos de aprendizagem e suas dificuldades.

Identificamos como sujeito de investigação e sua problemática: 03 trabalhos que trazem como tema o fracasso escolar, sendo 01 com crianças de classe popular pertencentes a uma Escola da Rede Estadual de Ensino localizada na periferia de Porto Alegre-RS. O trabalho utiliza-se de relato de crianças e de outros profissionais da escola, mas não identifica o número de pessoas investigadas. Em 02 outros trabalhos os sujeitos também são identificados como fracassados em sua escolaridade, sendo que uma pesquisa utiliza-se da análise de entrevistas realizadas com duas crianças e a outra é apresentada em forma de estudo de caso. Nos outros 05 trabalhos também apresentados como estudo de caso, encontramos as seguintes problemáticas: 01 criança com distúrbio no desenvolvimento global com suspeita de deficiência mental, diagnosticada por profissionais de diferentes áreas; crianças em atendimento psicomotor, porém através do resumo não foi possível identificar o número de crianças e a queixa específica; 01 criança que é apresentada como não sabendo ler aos oito anos e freqüentando a segunda série do Ensino Fundamental; 01 criança que é acompanhada na “sua travessia da inclusão”. Este resumo não relata a problemática específica da criança. Por fim, 01 criança que é identificada com severas dificuldades de aprendizagem na aquisição do código escrito, além de dificuldades relacionadas à linguagem oral. Há ainda, um trabalho de intervenção com 04 crianças identificadas como deficientes mentais.

O presente levantamento nos permite a constatação da complexidade do tema sobre a dificuldade de aprendizagem e os diversos fatores nela implicados. Entretanto não há produção significativa sobre aprendizagem fora do contexto de comprometimento orgânico, nem tampouco sobre a avaliação da própria criança sobre sua aprendizagem.

É importante destacar que não há uma homogeneidade nas informações dos resumos, dificultando uma análise mais sistemática e que nos permitisse a identificação dos objetivos, da metodologia utilizada, dos resultados e das conclusões. Dessa forma, optamos por uma análise mais geral dos trabalhos, destacando os aspectos considerados relevantes para a nossa temática.

Outros aspectos que consideramos significativos é que em 06 trabalhos também houve a participação de professores e outros profissionais da escola e/ou da família. Além disso, 
observamos que em 03 pesquisas, mesmo utilizando-se da criança como objeto de investigação, em suas conclusões apontam para a importância de trabalhos nesta área para auxiliar e fortalecer a função do educador e também a necessidade de se repensar e ressignificar o fracasso escolar.

Em alguns resumos também foi possível identificar a construção de instrumentos seja, para auxiliar o professor a entender e refletir sobre sua atuação em diferentes aspectos do seu trabalho: na relação com o aluno, no processo ensino-aprendizagem, além de propostas de intervenção com crianças com diferentes diagnósticos, mas que também enfrentam a problemática da dificuldade de aprendizagem. Observamos a utilização de diferentes abordagens face à psicanálise. Alguns explicitam os teóricos utilizados e outros trazem apenas os paradigmas utilizados. Dos autores da psicanálise que são citados encontramos Freud, Lacan, Melanie Klein. No que se refere à educação encontramos referência a Vigotsky. Também foram citados os paradigmas da Psicomotricidade Relacional, da Filosofia Benjaminiana, da Epistemologia Genética, e da Educação, da Educação Inclusiva e da Lingüística sem referências específicas aos autores utilizados.

É importante assinalar que quando utilizamos abordagens de diferentes correntes teóricas, numa perspectiva interdisciplinar, é altamente enriquecedor para o trabalho, porém Alves-Mazzotti (2002, p.32) nos alerta:

\begin{abstract}
A utilização de conceitos ou constructos pertencentes a teorias diversas para dar conta da complexidade dos fenômenos observados em um estudo requer cautela. Ao se valer de mais de uma vertente teórica para interpretar seus resultados, é necessário que o pesquisador esteja seguro de que as teorias utilizadas, das quais muitas vezes tomou apenas parte, não apresentem contradições entre seus pressupostos e relações.
\end{abstract}

Dentre os trabalhos analisados destacamos um que se aproxima mais da nossa temática por utilizar entrevistas com 02 crianças com histórico de fracasso escolar e que em suas conclusões considera que foi possível transformar a situação estudada, no caso, a da multirepetência, através dos caminhos apontados pelas próprias crianças. Compartilhamos desta perspectiva, por acreditar que possamos fazer o caminho inverso, ou seja, que ao trabalhar com a criança possamos tratar do caso em particular, mas que estes sejam estudos exemplares para ampliarmos a nossa atuação seja na família ou na escola.

Outros 03 trabalhos conforme observamos, também apresentam em seus resultados a importância de pesquisas nesta área no sentido de auxiliar e fortalecer a função do educador. 
Consideramos a relevância desses trabalhos, pois a criança está inserida numa rede de relações que não podem ser negadas ao estudar sua problemática, pois como já foi dito, a dificuldade de aprendizagem é multifatorial, portanto, seu caráter interdisciplinar. Muitas vezes a criança que apresenta dificuldade de aprendizagem é apenas o “porta voz” desta problemática.

Apesar de haver muitas pesquisas sobre a questão das dificuldades de aprendizagem e do fracasso escolar a partir de diferentes perspectivas, pouco se tem estudado ou considerado sobre o que pensam, sentem, desejam aqueles que sofrem com esta problemática. Em nossa experiência, junto ao atendimento psicopedagógico a crianças encaminhadas para o CENPE Centro de Pesquisas da Infância e da Adolescência “Dante Moreira Leite” - Unidade Auxiliar da Faculdade de Ciências e Letras - UNESP/Araraquara, com queixas de dificuldades de aprendizagem, encontramos crianças que nos chegam cheias de esperança de que finalmente alguém vai poder lhe ajudar, outras, no entanto, parecem já desacreditadas de que algo possa mudar.

Dessa forma, os dados obtidos nesta investigação nos incentivam ainda mais neste caminho, que é “escutar” as crianças que apresentam dificuldade de aprendizagem escolar, pois estas crianças já vivenciaram muitas situações de insucesso e podem ter muito a nos falar e como constatou o trabalho citado nos apontar caminhos para sua problemática em particular, mas também para a educação de forma geral. Esta é uma questão que nos acompanha em nosso atendimento psicopedagógico no CENPE e acreditamos esta pesquisa poderá trazer relevantes contribuições.

Ressaltamos a importância da pesquisa bibliográfica para nos auxiliar no encaminhamento do nosso trabalho, porém, a falta de homogeneidade da apresentação dos resumos, foi um fator dificultador na seleção dos mesmos.

Dos resultados obtidos, concluímos que a psicanálise tem sido utilizada enquanto teoria para compreender a problemática da educação e contribuir com novos olhares que considerem os aspectos psíquicos implicados nas relações entre os professores, entre alunos e professores, professores e demais profissionais da escola, entre os vários profissionais da escola e os pais dos alunos que de alguma forma enfrentam dificuldades em sua escolaridade, e também entre a escola e a sociedade, considerando os aspectos sócio-políticos, econômicos e culturais estabelecidas nesse campo.

A psicanálise a partir de seu referencial teórico e como método de investigação do psiquismo, tem contribuído para a compreensão dos fatores afetivos e relacionais implicados no ato de aprender e no processo de ensino-aprendizagem. No entanto, nossa educação 
costuma separar os domínios da vida afetiva e da vida cognitiva, como se ambas atuassem independentemente uma da outra, porém, sabemos da interdependência desses aspectos no processo de aprendizagem.

Concordamos com Assis e Oliveira (2003, p.246) quando escrevem que:

Para além dos métodos e técnicas, a aprendizagem se faz sempre na relação com outro, é fruto de relações que promovem o conhecimento e autoconhecimento. Acentuar a natureza da relação é ao mesmo tempo relativizar, a supremacia dos recursos da tecnologia pedagógica.

A psicanálise nos oferece instrumentos ao considerar a dimensão singular do sujeito aprendente e os aspectos inconscientes envolvidos no processo de aprendizagem.

Por fim, ressaltamos que as pesquisas que abordam a dificuldade de aprendizagem escolar e que focalizam o sujeito aprendente são em menor número e ainda pouco representativas diante da demanda que este tema traz atualmente. Dessa forma acreditamos que este trabalho poderá trazer contribuições relevantes para a interface psicanálise e educação de forma geral e mais especificamente para entender como se mostra o desejo de aprender/conhecer e o autoconceito que as crianças que passam por uma série de insucessos na escola têm sobre si mesmas.

\section{THE LEARNING DIFFICULTY IN SCHOOL UNDER THE OPTICS OF THE PSYCHOANALYSIS: AN INVESTIGATION OF THE SCIENTIFIC PRODUCTION}

ABSTRACT: This work presents an investigation of the current academic scientific production from 1996 to 2008 regarding the problems associated to the children with learning difficulties in Master's Project in School Education from the Faculdade de Ciências e Letras of UNESP/Araraquara, Brazil. The disabilities associated to learning difficulty were considered to be dependent on several factors (multifactorial nature), and the learning conditions were found to be intimately related to the psyche. The inspiring questions for the present research were the desire of knowledge/learning desire and the concept that children with learning difficulty have about themselves. Aiming at identifying what has been developed in this area, a search was carried out in the CAPES database by using the following keywords: psychoanalysis and school education within abstracts from Master and PhD theses. A number of 36 out of the 45 initially selected abstracts related to the search scope dealt with the theme of learning difficulty under the optics of the teacher, the social-politicaleducational questions, the programs dedicated to help children with special learning needs or theoretical aspects. Only nine abstracts included the child as the subject of the investigation; six abstracts dealt with case studies and three abstracts explored interviews and other resources to investigate what children enunciate about their disabilities. We concluded that the psychoanalysis has been used as a theory to deal with education and to contribute with new insights which take into account the psyquic aspects implicated in the stablished 
relations in this field. However, the research on the learning difficulty with focus on the learner is not so representative yet.

KEYWORDS: Psychoanalysis. Learning Difficulty. Child.

\section{REFERÊNCIAS}

ALVES-MAZZOTTI, A. J. A “revisão da bibliografia” em teses e dissertações: meus tipos inesquecíveis - o retorno. In: BIANCHETTI, L.; MACHADO A. M. N. (Org.). A bússola do escrever: desafios e estratégias na orientação de teses e dissertações. Florianópolis: Ed. da UFSC; São Paulo: Cortez, 2002. p.25-44.

ASSIS, M. B. A. C., OLIVEIRA, M. L. A psicologia do educador: a criança e o adolescente na atualidade. In: OLIVEIRA, M. L. (Org). Educação e Psicanálise: história, atualidade e perspectivas. São Paulo: Casa do Psicólogo, 2003. p. 239-254.

\section{BIBLIOGRAFIA CONSULTADA}

AZENHA, C. A. C. Lia: Do nome ao verbo ou desejo e leitura. 2006. 152p. Dissertação (Mestrado em Lingüística) - Instituto de Estudos da Linguagem, Universidade Estadual de Campinas, Campinas, 2006.

BATISTELA, L. F. Inibição intelectual e fracasso escolar: estudo de um caso. 1996. 102f. Dissertação (Mestrado em Educação) - Faculdade de Filosofia e Ciências, Universidade Estadual Paulista, Marília, 1996.

BOSSA, N. A. Fracasso escolar: um olhar psicopedagógico. Porto Alegre: Artmed, 2002.

BRAUNER, M. F. Escola: espaço de fabricação de imagem: Pátho(s) logias do olhar na relação professor-aluno. 2003. 350f. Tese (Doutorado em Educação) - Faculdade de Educação, Universidade Federal do Rio Grande do Sul, Porto Alegre, 2003.

COORDENAÇÃO DE APERFEIÇOAMENTO DE PESSOAL DE NÍVEL SUPERIOR [CAPES]. Banco de teses da CAPES. Brasília, 2008. Disponível em: $<$ http//serviços.capes.gov.br/capesdw>. Acesso em: 06 jul. 2009.

JORDÃO, M. C. M. A criança, a deficiência e a escola: uma intervenção orientada pela psicanálise. 2001. 225f. Dissertação (Mestrado em Psicologia Escolar e Desenvolvimento Humano) - Instituto de Psicologia, Universidade de São Paulo, São Paulo, 2001.

LIMA, S. O. Leitura e oralidade: as inscrições do desejo no percurso de formação do leitor. 2006. 236f. Tese (Doutorado em Educação) - Faculdade de Educação, Universidade de São Paulo, São Paulo, 2006. 
MASTRASCUSA, C. L. O que a criança nos diz quando parece nada falar? O

desbloqueio do discurso falado através do não-verbal. 2005. 138f. Dissertação (Mestrado em Educação) - Faculdade de Educação, Universidade Federal do Rio Grande do Sul, Porto Alegre, 2005.

SAMPAIO, W. M. Quando crianças falam a respeito do não-aprender: uma reflexão na interface da educação com a psicanálise. 1999. 220f. Dissertação (Mestrado em Educação) faculdade de Educação, Universidade Federal do Rio Grande do Sul, Porto Alegre, 1999.

SILVA, D. R. Q. "Fracasso" escolar: um lugar (re)pensado a partir de uma perspectiva psicanalítica. 2003. 112f. Dissertação (Mestrado em Educação) - Faculdade de Educação, Universidade Federal do Rio Grande do Sul, Porto Alegre, 1999.

TOMEDI, J. Psicanálise e educação: inclusão, os caminhos do sujeito na perspectiva da diferença. 2007. 80f. Dissertação (Mestrado em Educação) - Faculdade de Educação, Universidade Federal do Rio Grande do Sul, Porto Alegre, 2007 